Editorial

(

\title{
Advances in rapid pathogen detection
}

\section{Introduction}

Microbiological analysis has progressed considerably in the twentyfirst century through the advent of rapid microbiological methods. This is most apparent within the clinical, ${ }^{1}$ and pharmaceutical sectors. ${ }^{2}$ One area of much needed advancement is with pathology. Here clinical investigators can take samples from the field, from patients or from the environment, and undertake rapid analysis, obtaining results within hours rather than the days or weeks required with conventional methods. Time is often a consequence of slow-growing, difficult to culture, and unculturable microorganisms. Time-to-result is critical when making decisions about patient health. In this editorial, some of the most recent advances with rapid methods for pathogen detection are considered.

\section{Emerging rapid microbiological methods}

Rapid (and alternative) microbiological methods are ambiguous terminology, since "rapid" is open to interpretation and requires a new method to be considered relative to an established one. Moreover, rapid methods are invariably presumed to be more accurate, although this is not always the case. Rapid methods cover a spectrum from miniaturized biochemical kits; antibody tests; DNA-based tests; as well as culture-based tests that are modifications of conventional tests, such as the examination of 'micro-colonies' using light scanning.

In terms of assessing pathologies, clinical areas where rapid methods are an advantage include areas where urgent (and correct) decision making is required. Examples include: pneumonia in the immunocompromised; bacteremia; meningitis; soft tissue infection; eye disease; endocarditis and so on. ${ }^{3}$

One such example is with the clinical decision as to which antimicrobial a patient requires in response to a bacterial infection. Administering the incorrect antimicrobial has consequences for the patient, and arguably contributes to the problem of emerging antimicrobial resistance. ${ }^{4}$ To allow the clinician to take a sample, obtain a result, and decide on the appropriate antimicrobial inside of one hour, University of Toronto researchers have designed a prototype chip that can rapidly test for antibiotic resistance through phenotyping. The process works by concentrating any bacteria extracted from a patient blood sample into a compact space inside microfluidic wells (two nanoliters in volume). This tony space allows the volume to be increased and, in effective, increases concentration of the starting sample. Inside each well a lattice of microbeads is located. These serve to capture bacteria as a sample is passed through. Captured bacteria accumulate in a well into which a range of antimicrobials are placed together resazurin, which functions as an electrochemical signature molecule through conversion to resorufin. ${ }^{5}$

As an alternative, C-reactive protein (CRP) blood tests have been demonstrated as capable of showing if an infection is bacterial. The method determines the level of C-reactive protein present in the blood; these levels correlate with levels of inflammation present in the body. ${ }^{6}$

In addition to determining the type of organism quantifying the numbers is an additional part of clinical assessment. Classic methods, such as haemocytometer or Neubauer chamber, take time to analyse. A faster method that has been explored is with the use of

Volume 2 Issue 5 - 2015

\author{
Tim Sandle \\ Pharmig (Pharmaceutical Microbiology Interest Group), \\ Stanstead Abbotts, UK
}

Correspondence: Tim Sandle, Pharmig (Pharmaceutical Microbiology Interest Group), Stanstead Abbotts, UK, Email timsandle@btinternet.com

Received: November 03, 2015 | Published: November 05, 2015

spectrophotometry to measure the absorbance of a cell suspension. As an example, researchers have reported the success of using this method for assessing numbers of the fungus Fusarium, counting values for conidia by concentration. ${ }^{7}$

With viruses, a current area of epidemiological concern is with avian influenza. Here University of Guelph scientists have worked on a novel biosensor that can characterise an influenza virus in around three minutes from blood samples. The technique is based on gold nanoparticles that enable the surface proteins found on viral particles to be detected via nanobiosensor. Here different colours signal the presence of a virus and can sig different types of avian flu. Trials have been successfully undertaken at poultry farms. ${ }^{8}$ Biosensors are also being applied to antimicrobial drug discovery. Sensors can be used to screen a range of candidate drugs by obtaining of MIC (Minimum Inhibitory Concentration) values. ${ }^{9}$

In terms of environmental screening, a research group from Ben-Gurion University of the Negev and the Massachusetts Institute of Technology have used qPCR (quantitative polymerase chain reaction) to examine samples of water and soil. With qPCR, screening of pathogens is completed within 24 hours. qPCR looks at the amplification of DNA, through the generation of thousands to millions of copies of a particular DNA sequence, in real time and can detect down to one microbial cell. ${ }^{10}$ This allowed a limit of detection of down to one cell to be achieved. In trials, the research group successfully identified a specific strain of Salmonella (S. enterica), selected from soil samples, and Pseudomonas aeruginosa from samples of water. ${ }^{11}$ In terms of patient samples, real-time quantitative PCR has been successfully deployed to detect multidrug-resistant tuberculosis within one hour. ${ }^{12}$

With microbial identification, MALDI-TOF (Matrix-Assisted Laser Desorption/Ionization-Time Of Flight) mass spectrometry has emerged as an adjunct test between slower, culture-based phenotypic methods and genotypic methods. ${ }^{13}$ With MALDI-TOF it is possible to detect the bacteria in a sample in less than three minutes, with good specificity (depending on the scope of the system database.)

\section{Summary}

The development and adoption of rapid microbiological methods comes with several advantages for improvements to the quality of patient care. These are with faster diagnosis and more accurate assessment of pathological conditions. Over the longer-term, these 
improved assessments will help to reduce patient health care costs and enable more effective patient management. Thus, it is to such technologies that we should be focusing on in the context of modern microbiology and experimentation.

\section{Acknowledgments}

None.

\section{Conflicts of interest}

Author declares that there is no conflict of interest.

\section{References}

1. Frickmann H, Masanta WO, Zautner AE. Emerging rapid resistance testing methods for clinical microbiology laboratories and their potential impact on patient management. Biomed Res Int. 2014;2014:375681.

2. Sandle T, Leavy C, Jindal H, et al. Application of rapid microbiological methods for the risk assessment of controlled biopharmaceutica environments. J Appl Microbiol. 2014;116(6):1495-1505.

3. Jorgensen JH. Rapid methods in clinical microbiology. Clinical situations in which rapid methods may best be applied. Diagn Microbiol Infect Dis. 1985;3(6 Suppl):3S-7S

4. Sandle T. The antibiotic crisis and the need for new forms of antibiotics. EC Microbiology. 2014;1(1):1-3.

5. Besant JD, Sargent EH, Kelley SO. Rapid electrochemical phenotypic profiling of antibiotic-resistant bacteria. Lab Chip. 2015;15(13):2799-2807.

6. Lelubre C, Anselin S, Zouaoui Boudjeltia K, et al. Interpretation of $\mathrm{C}$-reactive protein concentrations in critically ill patients. Biomed Res Int. 2013;2013:124021.

7. Lage L, Panizo MM, Ferrara G, et al. Validation of the inoculum by densitometry for antifungal susceptibility testing in Fusarium species. Rev Soc Ven Microbiol. 2013;33(1):46-52.

8. Mungroo NA, Neethirajan S. Biosensors for the detection of antibiotics in poultry industry-a review. Biosensors (Basel). 2014;4(4):472-493.

9. van Belkum A, Dunne WM. Next-generation antimicrobial susceptibility testing. J Clin Microbiol. 2013;51(7):2018-2024.

10. Sandle T, Azizov I, Babenko D, et al. Comparative evaluation of traditional susceptibility testing for MRSA with the PCR approach. Advances in Microbiology. 2014;4:1204-1211.

11. Orlofsky E, Benami M, Gross A, et al. Rapid MPN-Qpcr screening for pathogens in air, soil, water, and agricultural produce. Water, Air \& Soil Pollution. 2015;226:303.

12. Ramirez MV, Cowart KC, Campbell PJ, et al. Rapid detection of multidrug-resistant Mycobacterium tuberculosis by use of realtime PCR and high-resolution melt analysis. J Clin Microbiol. 2010;48(11):4003-4009.

13. Sandle T.Automated microbial identifications: A comparison of USP and EP approaches. American Pharmaceutical Review. 2013;16(4):56-61. 